\title{
Albumin is not suitable as a marker of bronchoalveolar lavage dilution in interstitial lung disease
}

\author{
C. Ward*, J. Fenwick**, H. Booth*, E.H. Walters*
}

\begin{abstract}
Albumin is not suitable as a marker of bronchoalveolar lavage dilution in interstitial lung disease. C. Ward, J. Fenwick, H. Booth, E.H. Walters. @ERS Journals Ltd 1997. ABSTRACT: We have investigated the origin of raised bronchoalveolar lavage (BAL) albumin levels in interstitial lung diseases (ILDs), and the hypothesis that acute flux during BAL might contribute to the elevated levels of albumin in samples from ILD patients.

Total albumin concentrations were measured in three separately aspirated 60 $\mathrm{mL}$ aliquots of BAL in 12 ILD patients and seven control subjects, and previous work indicating that BAL albumin levels may be raised in ILD was confirmed. In addition, the extent of acute flux from the circulation was investigated using 1.48 $\mathrm{MBq}$ of $125 \mathrm{I}$-radiolabelled human serum albumin injected intravenously shortly before the procedure.

It was found that acute albumin flux occurred in only a minority of the ILD patients (4 out of 12) and control subjects ( 3 out of 7$)$. These results indicate that the elevated levels of plasma proteins, such as albumin, documented in BAL aspirates from ILD patients are not an artefact of the BAL procedure related to increased vascular permeability, and that this finding is more likely to be related to the effects of chronic inflammatory changes in the lung due to the underlying disease.

The work confirms that albumin is unsuitable as a denominator for the normalization of bronchoalveolar lavage solute results, since levels can vary, both as a result of acute flux and, more frequently, because of disease activity. Eur Respir J 1997; 10: 2029-2033.
\end{abstract}

*Dept of Respiratory Medicine, Alfred Hospital and Monash University Medical School, Melbourne, Australia. **Medical Physics Dept, Newcastle General Hospital, Newcastle upon Tyne, UK.

Correspondence: E.H. Walters

Dept of Respiratory Medicine

Alfred Hospital

Commercial Rd

Prahran

Melbourne

Victoria 3181

Australia

Keywords: Albumin

bronchoalveolar lavage

dilution

interstitial lung disease

permeability

Received: October 211996

Accepted after revision February 131997

Supported, in part, by the NHMRC of Australia, the Alfred Hospital Research Foundation and Glaxo Group Research and Development.
The realization that changes in microvascular permeability may be an important contributory factor to the pathophysiology of inflammatory airway disease [1] has led to development of animal models to study such changes. Approaches that have been used include the measurement of total albumin or protein in the lung. This has been complemented in some studies by the monitoring of extravasation of indirect tracers of plasma proteins, such as Evans blue or Monastral blue [1,2], which have the common property of binding to serum albumin in a manner that can be subsequently quantified. This type of experimental design has been used to examine the effect of anti-inflammatory drugs [3].

There is increasing evidence that local plasma leakage due to changes in microvascular permeability occurs in human inflammatory airway and lung diseases. This has been suggested as the cause of elevated levels of bronchoalveolar lavage (BAL) protein in patients with different forms of interstitial lung disease (ILD) $[4,5]$, especially sarcoidosis and extrinsic allergic alveolitis [6], but also following airway challenge in asthmatics $[7,8]$.

BAL, however, is a complex process [9], with the procedure itself influencing components of the aspirate. Large bidirectional movements of water have been shown to occur between the instilled BAL fluid and the circulation [10], with a large net gain to the BAL fluid, probably due to application of suction pressure to the system. The finding that urea $[11,12]$ sampled at BAL increased in amount with dwell time between the instillation and aspiration of the BAL fluid indicated that low molecular weight solutes are also likely to move acutely from blood to BAL fluid, following their concentration gradient.

Furthermore, in an earlier study, in which we investigated the origin of water and urea sampled at BAL in asthmatic and control subjects using radiolabelled tracers, we found a strong relationship between influx of radiolabelled water and radiolabelled urea from blood to BAL fluid, suggesting that urea sampled at BAL may have been derived predominantly from an acute movement from the circulation into the BAL aspirate accompanying the large movement of water [13]. These findings imply that the elevated levels of larger molecular weight solutes, such as albumin, or total protein in BAL fluid, found in certain conditions might not be a simple function of local inflammation, but might also reflect an interaction between the sampling method used and an altered microvasculature.

In a previous study [14], we developed a system which allowed monitoring of albumin flux in human subjects undergoing BAL procedures using small amounts of an intravenously administered radiolabelled tracer (1.48 
$\mathrm{MBq}{ }^{125}$ I-labelled human serum albumin). Using this approach, together with the analysis of total BAL albumin levels, we have now sought to evaluate the origin of BAL albumin in ILD. This is of particular relevance, since BAL albumin has frequently been suggested as a means of "normalizing" the concentration of other BAL solutes between individuals and groups [15-17]. It is perhaps surprising that despite the fact that measurement of BAL solutes is increasingly used as an endpoint in clinical research, there remains little documented work in human subjects evaluating their origin compared with extensive work in animal models and isolated lung preparations [18-20].

In this study, we have investigated whether elevated levels of BAL albumin in ILD are a direct marker of lung inflammation or are secondary to increased acute flux from the circulation through a more permeable vascular-endothelial/epithelial membrane. The study involved measuring total BAL albumin levels in patients with ILD and in controls, with the contribution of acute flux from the circulation to the overall level of BAL albumin being estimated from radiolabelled albumin injected into the circulation 5 min prior to BAL.

\section{Methods}

Ethics permission for the study was obtained from the local Joint Ethics Committee.

\section{Subject groups}

The 12 subjects with ILD were volunteers, with a well-documented active disease (table 1). The seven control subjects were undergoing diagnostic bronchoscopy, mainly for an episode of minor haemoptysis for which no cause was evident. They had normal lung function, no evidence of general airway or pulmonary disease and no local abnormality at the site lavaged.

\section{Physiology}

Spirometry was carried out on all subjects using a wedge bellows spirometer (Vitalograph, Buckingham,

Table 1. - Demographic details of the 12 ILD patients and seven control subjects in the study

\begin{tabular}{lcc}
\hline & ILD & Controls \\
\hline Subjects n & 12 & 7 \\
Age yrs & & $54(27-72)$ \\
Smokers n & $123-71)$ & 1 \\
FEV $\%$ pred & $88(43-115)$ & $94(70-126)$ \\
TL,CO,sb \% pred & $69(24-109)$ & $98(86-111)$ \\
Sex M/F & $8 / 4$ & $4 / 3$ \\
Diagnoses & Sarcoid 7 & Normal \\
& IPF 2 & \\
& EAA 2 & \\
& BOOP 1 &
\end{tabular}

\#: median, and range in parenthesis; $¥$ : mean, and range in parenthesis. ILD: interstitial lung disease; FEV1: forced expiratory volume in one second; $\%$ pred: percentage of predicted value; $T \mathrm{~L}, \mathrm{CO}, \mathrm{sb}$ : single-breath transfer factor of the lung for carbon monoxide; IPF: idiopathic pulmonary fibrosis (cryptogenic fibrosing alveolitis (CFA)); EAA: extrinsic allergic alveolitis; BOOP: bronchiolitis obliterans organizing pneumonia.
UK). In addition, the single-breath transfer factor of the lung for carbon monoxide $(T \mathrm{~L}, \mathrm{CO}, \mathrm{sb})$ was measured (Autolink; P.K. Morgan Ltd, Rainham, UK).

\section{Assessment of albumin flux and BAL details}

Five minutes before BAL, $1.48 \mathrm{MBq}$ of ${ }^{125}$ I-labelled human serum albumin in $0.5 \mathrm{~mL}$ of normal saline was injected intravenously into an anterior cubital fossa vein.

BAL was performed in three $60 \mathrm{~mL}$ aliquots using sterile, pyrogen-free, phosphate-buffered saline, prewarmed to $37^{\circ} \mathrm{C}$, instilled into a segment of the right middle lobe, using an Olympus BF P10 bronchoscope (5 mm diameter; Olympus, Tokyo, Japan). Aspiration of fluid was performed at a pressure of -50 to -100 $\mathrm{mmHg}$ into separate, iced, siliconized glass vessels.

BAL total cell counts were made on mixed, unprocessed BAL fluid, for the three separate aliquots using a modified Neubaur counting chamber. In addition, in the ILD patients, aliquots of unprocessed BAL were reserved in order to perform a pooled BAL cell differential. Differential cell counts were made on duplicate cytospin preparations (Shandon cytospin II, $500 \mathrm{rpm}, 2$ min; Shandon, Runcorn, Chesire, UK), by two observers count-ing 1,000 cells each.

Cells were separated from BAL supernatants within $1 \mathrm{~h}$ by centrifugation for $15 \mathrm{~min}$ at $100 \times \mathrm{g}$. Each BAL supernatant was subdivided into $4 \mathrm{~mL}$ aliquots and stored at $-80^{\circ} \mathrm{C}$ until subsequent biochemical assays.

\section{Isotope counting}

Gamma counts for ${ }^{125}$ I were made on aliquots taken from the three separate BAL supernatants and on venous blood plasma samples taken simultaneously from the arm contralateral to that used for the isotope injection. A count time of $60 \mathrm{~min}$ was used. Counts were corrected for background levels, assessed using a blank tube. It was planned to exclude any subject with frank blood-staining of the BAL aspirate, but fortunately, in the event, this was not necessary.

\section{Albumin assays}

Albumin concentrations in unconcentrated BAL supernatants were measured using a COBAS FARA II autoanalyser (Roche Diagnostics Ltd, Basel, Switzerland). Plasma albumin levels were measured using a Hitachi 717 (Tokyo, Japan) continuous flow autoanalyser.

\section{Calculations}

The amount of BAL albumin derived acutely by influx from the circulation into BAL fluid ("A") was calculated by proportionality:

$$
\text { "A" }=\frac{\mathrm{cpm} \cdot \mathrm{mL}^{-1} \mathrm{BAL}}{\mathrm{cpm} \cdot \mathrm{mL}^{-1} \text { plasma }} \times(\text { plasma albumin })
$$

Where $\mathrm{cpm} \cdot \mathrm{mL}^{-1}$ represents the gamma counts in counts per minute per millilitre of BAL supernatant or plasma. 


\section{Statistical analysis}

Statistical analysis was performed using the statistical package Minitab (CLE COM Ltd, Birmingham, UK). Comparisons of BAL albumin levels between groups were made using the unpaired t-test, and comparisons within groups were made using the paired t-test. In order to investigate potential relationships between BAL inflammatory cell counts and albumin levels, a limited number of Pearson correlation analyses were performed in the ILD subjects. The analyses were made on ranked data, in order to prevent undue influence from outliers, and a limited number of end-points were prospectively defined. A p-value of less than 0.05 was considered to be significant.

\section{Results}

The dilution volumes for the injected ${ }^{125}$ I-labelled albumin were within predicted values of plasma volumes in all subjects. This indicated that there was no significant movement of albumin from the circulation into the extracellular space by the time of BAL.

The total BAL albumin concentrations assayed, in $\mu \mathrm{g} \cdot \mathrm{mL}^{-1}$ of return, are summarized in figure $1 \mathrm{a}$ for the separate aliquots in the subjects studied. Overall there were significantly greater levels of albumin detected in BAL fluid from the population of patients with ILD when compared with controls, although there was a wide range. The differences in albumin concentrations between the two groups were most marked in the first and second BAL aliquots.

BAL differential cell counts for the pooled data from the ILD subjects are shown in table 2, together with our previously published laboratory normal range [21] for comparison. A formal, statistical comparison of cell differentials in this mixed population of ILD versus controls was not an end-point of this study, however, the differential cell counts were similar to previous findings in ILD, in that they were consistent with an elevated total cell count and increased numbers of lymphocytes and granulocytes.

Prospectively, we designated potential relationships between total albumin return and total absolute cell return, total absolute lymphocyte return and total absolute granulocyte (i.e. neutrophils and eosinophils) return, as end-points for correlation analyses. There were no significant relationships detected between total albumin return and total absolute cell returns ( $\mathrm{r}=0.336$; Ns), total absolute lymphocyte returns ( $\mathrm{r}=0.455$; Ns), or total absolute granulocyte returns ( $\mathrm{r}=-0.477$; NS).

The majority of the patients and controls exhibited either no or minimal levels of acute albumin flux from the circulation into the BAL aspirates, with no significant difference between the groups $(\mathrm{p}=0.991)$ (fig. 1b). However, in a small number, a large amount of flux occurred, and on one occasion all the albumin measured in the second aliquot from an ILD subject was accounted for by acute flux. Overall, in the patients where flux occurred, there was no relationship between absolute albumin levels and the amount of albumin flux observed ( $r=-0.397$; NS).

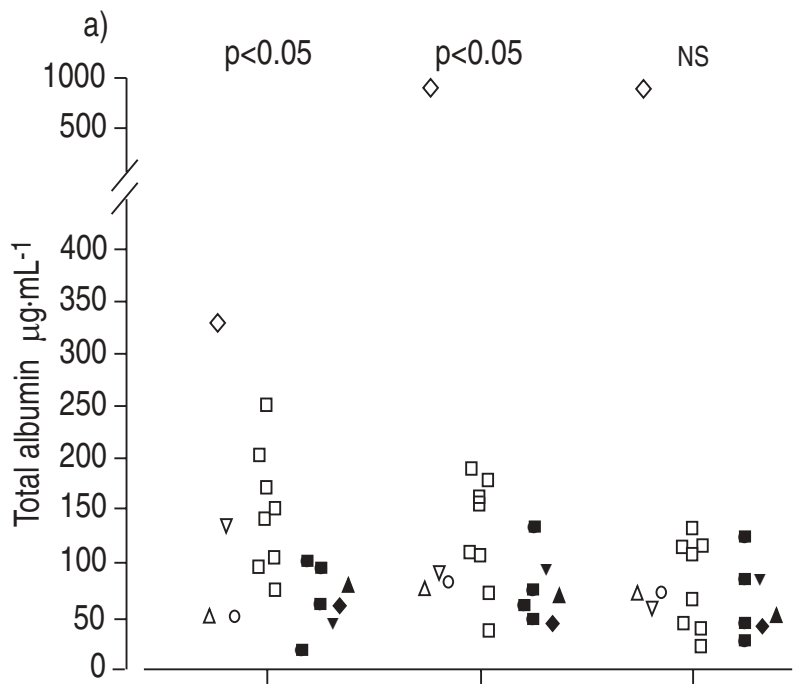

b)

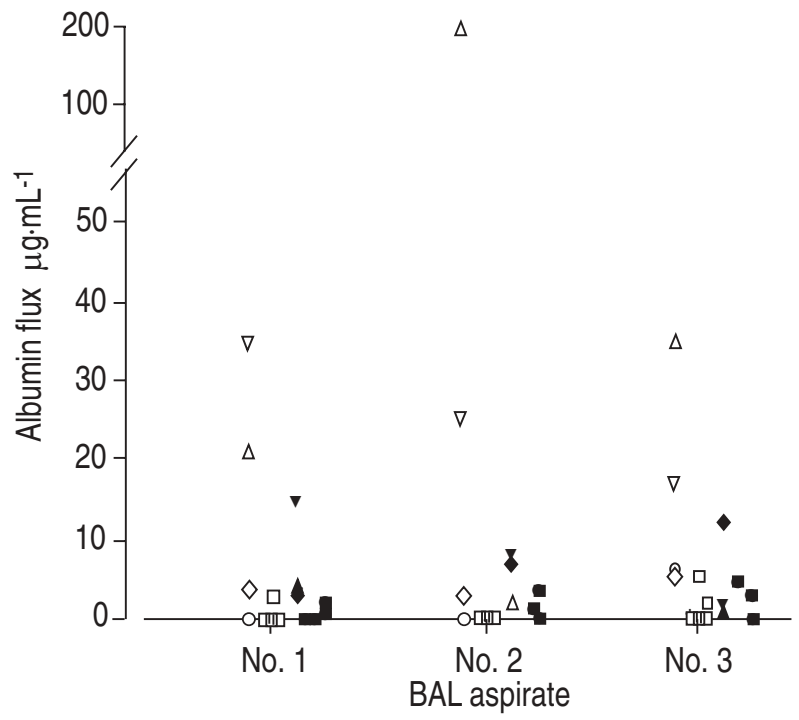

Fig. 1. - a) Total albumin measured directly in the three aliquots of BAL aspirate (Nos. 1-3); and b) the albumin flux calculated to have occurred into aspirates by radiolabelling. ILD patients are represented by open symbols and the controls by closed symbols. Where no significant flux was detected, subjects are denoted by squares, and where a significant flux was detected, by other symbols, to allow cross referencing of total albumin measurements and albumin flux within individuals. Where quoted, p-values relate to comparisons of ILD and control subjects. BAL: bronchoalveolar lavage; ILD: interstitial lung disease.

Table 2. - BAL differential cell counts for the pooled data from the 12 ILD subjects, together with our previously published laboratory normal range based on 27 normal subjects [21]

\begin{tabular}{lcccccc}
\hline & $\begin{array}{c}\text { TCC } \\
\times 10^{6}\end{array}$ & $\begin{array}{c}\text { AM } \\
\%\end{array}$ & Lym & Eos & PMN & Epi \\
\hline ILD & 24 & 57 & 35 & 1 & 3 & 0.3 \\
& $(13-84)$ & $(16-77)$ & $(15-83)$ & $(0-21)$ & $(0-23)$ & $(0-10)$ \\
Normals & 18 & 79 & 17 & 0.3 & 2 & 0.6 \\
& $(4-41)$ & $(51-95)$ & $(3-46)$ & $(0.1-4.2)$ & $(0.1-7)$ & $(0.2-3)$ \\
\hline
\end{tabular}

Values are presented as median, and range in parenthesis. ILD: interstitial lung disease; TCC: total cell count in BAL return; AM: alveolar macrophages; Lym: lymphocytes; Eos: eosinophils; PMN: polymorphonucleates; Epi: epithelial cells. 


\section{Discussion}

The results of this study confirm previous work, which has demonstrated elevated albumin levels in BAL fluid from subjects with ILD [4], especially subjects with granulomatous diseases, such as sarcoidosis [5]. We have extended the work by investigating the source of this elevated BAL albumin using radiolabelled human serum albumin as a tracer. In particular, we investigated the hypothesis that acute movement of plasma proteins from the circulation, induced by the BAL procedure itself, might have contributed to the elevated levels of BAL albumin, as well as chronic leakage due to the inflammatory process underlying ILD.

Our hypothesis was formulated in view of our earlier studies, which have shown large movements of labelled water and urea during the procedure of BAL $[10,13]$, and on the basis of work in animal models, which have demonstrated that local distortion of tissue due to high hydrostatic pressures can lead to the opening of nonphysiological pores in the epithelial/endothelial barrier [18, 19]. Hence, the relatively large and unphysiological aspiration pressures applied during BAL, to what is normally a low pressure system, might represent a mechanism causing acute movement of plasma proteins during BAL. Potentially, such a process could be exacerbated when the blood to alveolar membrane is already more permeable because of inflammation. It is, perhaps, surprising that despite the fact that BAL fluid solutes are commonly measured in human clinical research, there is very little published work that has sought to evaluate their origins, i.e. local production, chronic leakage from the blood, or acute movement from the blood during BAL due to an increased blood/alveolar permeability.

The results of our study indicated that acute flux of albumin from the circulation into BAL fluid occurred in only a minority of the ILD patients (4 of the 12 studied) and control subjects ( 3 of the 7 studied). The absolute amount of flux in each BAL aliquot for these subjects tended to be greater for the ILD patients, but not significantly so. For the patients where flux occurred, there was no correlation between total albumin levels and the amount of albumin flux observed.

The observation that acute movement of albumin occurred in only a minority of the ILD patients and the controls, in the context of a BAL protocol involving immediate aspiration, leads us to speculate that unphysiological suction pressures generated during the process of BAL, interacting with a variably permeable vascular/alveolar barrier, were responsible for our observations, since a mechanism involving purely diffusion along concentration gradients would, presumably, have been more uniformly observed. The application of negative pressure during BAL aspiration is rather imprecise, with little control over the surface area to which that pressure is applied. There was no obvious technical difference in the BAL procedures where flux was observed, though presumably local distortion may have caused strong, relatively focal negative pressure. Alternatively, in the individuals where flux occurred, the barrier between blood and airway may have been particularly permeable because of a different, or more aggressive, disease process.
In one of the ILD subjects studied, the amount of albumin calculated to be derived from the circulation by radioisotope labelling in the second aliquot was equal to or even greater than the amount actually assayed directly in the BAL supernatant. This individual result from the 57 such sets of measurements made in the study may have been a technical artefact, but the result has not been censored, in order to retain complete data. The large differences between plasma and BAL concentrations of albumin mean that the use of an indirect tracer to make estimations of BAL albumin is likely to be less accurate than direct biochemical assays, but, in general, we feel that our methodology was robust enough to give an appropriate estimate of albumin flux and we have confidence in the general conclusions of the study. We feel that this type of approach is useful in studies aimed at evaluating BAL methodology, but that it is probably too involved for use in routine BAL procedures.

The primary end-point of this study was to investigate the origin of the raised BAL albumin levels previously described in ILD [4-6]. Our results indicated that this is not an artefact of the BAL procedure, and is more likely to be related predominantly to the effects of chronic inflammatory changes in the lung due to the underlying disease.

We also found, however, that there were no simple relationships between BAL inflammatory cell numbers and albumin levels, as denoted by univariate correlation analyses, in the ILD patients studied. Hence, elevated levels of BAL albumin alone are not likely to be useful in the clinical assessment of ILD disease "activity", since this is likely to be a multifactorial and complex process. However, it is one factor, among many, to be taken into account in the assessment of ILD.

It should be noted that this study again underlined the complex nature of BAL sampling. In particular, BAL does not take place in a closed system of an intubated subsegment of the bronchoalveolar tree. The BAL sampling method potentially distorts the microvascular circulation of the lung and the barrier between the blood and air, both through physical trauma and the creation of diffusion gradients, and this is likely to vary substantially between individuals for purely technical reasons that cannot easily be quantified. This may be particularly important in the case of small molecular weight solutes, but it is clear that such considerations should be borne in mind by all groups interpreting the measurements of BAL solute levels, especially in the light of emergent interest in this area, with assays of cytokines [22], soluble adhesion molecules [23], and soluble markers of cellular activation in BAL fluid becoming technically routine [21, 24].

The work also underlines earlier studies by our group [14] and others [5] that have shown that BAL albumin or total protein levels are unsuitable for use as denominators to allow for variable dilution of epithelial lining fluid (ELF), although this is still being done or proposed [15-17]. This would include the types of study that have calculated "distribution coefficients" for BAL solutes, by relating ratios of BAL and serum measurements of solutes, with albumin being used as a denominator $[25,26]$. There is now a wide body of literature indicating that BAL albumin or total protein can change 
independently of ELF volume. Potential mechanisms for this include acute flux in some individuals, but more important are local effects of the disease itself, a process that will vary, either spontaneously, or as a result of antiinflammatory treatment. Furthermore, alternative approaches, such as the urea method for determining ELF volume, cannot be recommended [13]. For these reasons, we would support the view that the expression of measurements of BAL solute data is most meaningful when the results are expressed per millilitre of bronchoalveolar lavage aspirate, or as total bronchoalveolar lavage return.

Acknowledgements: The authors gratefully acknowledge the assistance of M. Duddridge and P.V. Gardiner in patient assessment and bronchoscopy, and A. Weddle with albumin measurements.

\section{References}

1. Chung KF, Rogers DF, Barnes PJ, Evans TW. The role of increased airway microvascular permeability and plasma exudation in asthma. Eur Respir J 1990; 3: 329-337.

2. Yoshihara S, Chan B, Yawawaki I, et al. Plasma extravasation in the rat trachea induced by cold air is mediated by tachykinin release from sensory nerves. Am J Respir Crit Care Med 1995; 151: 1011-1017.

3. Whelans CJ, Johnson M. Inhibition by salmeterol of increased vascular permeability and granulocyte accumulation in guinea-pig lung and skin. Br J Pharmacol 1992; 105: 831-838.

4. Reynolds HY. Bronchoalveolar lavage. Am Rev Respir Dis 1987; 135: 250-263.

5. Jones KP, Edwards JH, Reynolds SP, Peters TJ, Davies BH. A comparison of albumin and urea as reference markers in bronchoalveolar lavage fluid from patients with interstitial lung disease. Eur Respir J 1990; 3: 152-156.

6. Schmekel B, Wollmer P, Venge P, Linden M, BlomBulow B. Transfer of 99mTc-DTPA and bronchoalveolar lavage findings in patients with asymptomatic extrinsic allergic alveolitis. Thorax 1990; 45: 525-529.

7. Lam S, Leriche JC, Kijek K, Phillips D. Effect of bronchial lavage volume on cellular and protein recovery. Chest 1985; 88: 856-859.

8. Fick RB, Metzger JW, Hal RB, et al. Increased bronchovascular permeability after allergen exposure in sensitive asthmatics. J Appl Physiol 1987; 63: 1147-1155.

9. Task Group Report. Technical recommendations and guidelines for bronchoalveolar lavage (BAL). Eur Respir $J$ 1989; 2: 561-585.

10. Kelly CA, Fenwick JD, Corris PA, Fleetwood A, Hendrick DJ, Walters EH. Fluid dynamics during bronchoalveolar lavage. Am Rev Respir Dis 1988; 138: 81-84.

11. Marcy TW, Merril WW, Rankin JA, Reynolds HY. Limitations of using urea to quantify epithelial lining fluid recovered by bronchoalveolar lavage. Am Rev Respir Dis 1987; 135: 1276-1280.
12. Schmekel B, Venge P. The distribution of myeloperoxidase, eosinophilic cationic protein, albumin and urea in sequential bronchoalveolar lavage. Eur Respir J 1991; 4: 517-523.

13. Ward C, Duddridge M, Fenwick J, et al. Urea as a dilution marker for the determination of the epithelial lining fluid at bronchoalveolar lavage. Am Rev Respir Dis 1992; 146: 444-447.

14. Ward C, Duddridge M, Fenwick J, et al. An evaluation of albumin as a reference marker for bronchoalveolar lavage fluid in asthmatic and control subjects. Thorax 1993; 48: 518-522.

15. Low RB, Davis GS, Giancola MS. Biochemical analyses of bronchoalveolar lavage fluid of healthy volunteer smokers and nonsmokers. Am Rev Respir Dis 1978; 118: 863-875.

16. Merrill W, O'Hearn E, Rankin J, Naegel G, Matthay RA, Reynolds HY. Kinetic analysis of respiratory tract proteins recovered during a sequential lavage protocol. Am Rev Respir Dis 1982; 126: 617-620.

17. Bridgeman MME, Marsden M, MacNee W, Flenley DC, Ryle AP. Cysteine and glutathione concentrations in plasma and bronchoalveolar lavage fluid after treatment with N-acetylcysteine. Thorax 1991; 46: 39-42.

18. Wright EM. Solute and water transport across epithelia. Am Rev Respir Dis 1983; 127: S3-S8.

19. Egan EA. Fluid balance in the air-filled alveolar space. Am Rev Respir Dis 1983; 127: S37-S39.

20. Effros RM, Chang HK (Eds). In: Fluid and solute transport in the airspaces of the lungs. New York, Marcel Dekker, 1994; pp. 375-397.

21. Booth H, Richmond I, Ward C, Gardiner PV, Harkawat R, Walters EH. Effect of high-dose inhaled fluticasone propionate on airway inflammation in asthma. Am J Respir Crit Care Med 1995; 152: 45-52.

22. Chollet-Martin S, Gatecel C, Kermarrec N, GougerotPocidalo MA, Payen DM. Alveolar neutrophil functions and cytokine levels in patients with adult respiratory distress syndrome during nitric oxide inhalation. Am J Respir Crit Care Med 1996; 153: 985-990.

23. Zangrilli JG, Shaver JR, Cirelli RA, et al. sVCAM-1 levels after segmental antigen challenge correlate with eosinophil influx, IL-4 and IL-5 production, and the late phase response. Am J Respir Crit Care Med 1995; 151: 1346-1353.

24. Striz I, Zheng L, Wang YM, Pokorna H, Bauer PC, Costabel U. Soluble CD14 is increased in bronchoalveolar lavage of active sarcoidosis and correlates with alveolar macrophage membrane-bound CD14. Am J Respir Crit Care Med 1995; 151: 544-547.

25. Holter JF, Weiland JE, Pacht ER, Gadek JE, Davis WB. Protein permeability in the adult respiratory distress syndrome: loss of size selectivity of the alveolar epithelium. J Clin Invest 1986; 78: 1513-1522.

26. Krsek-Staples JA, Kew RR, Webster RO. Ceruloplasmin and transferrin levels are altered in serum and bronchoalveolar lavage fluid of patients with the adult respiratory distress syndrome. Am Rev Respir Dis 1992; 145: 1009-1015. 